\title{
DMS advanced applications for accommodating high penetrations of DERs and microgrids
}

\author{
Arindam Maitra ${ }^{凶}$, Tanguy Hubert ${ }^{1}$, Jianhui Wang ${ }^{2}$, Ravindra Singh ${ }^{2}$, \\ Ning Kang ${ }^{1}$, Xiaonan Lu ${ }^{2}$, Jim Reilly ${ }^{3}$, Annabelle Pratt ${ }^{4}$, Santosh Veda ${ }^{4}$ \\ ${ }^{1}$ Electric Power Research Institute, Palo Alto, CA, USA \\ ${ }^{2}$ Argonne National Laboratory, Illinois, USA \\ ${ }^{3}$ Reilly Associates, Stroudsburg, PA, USA \\ ${ }^{4}$ National Renewable Energy Laboratory, Denver, CO, USA \\ 凶-mail: amaitra@epri.com
}

\begin{abstract}
Efficient and effective management of the electric distribution system requires an integrated approach to allow various systems to work in harmony, including distribution management systems (DMS), distributed energy resources (DERs), distributed energy resources management systems, and microgrids. This study highlights some outcomes from a recent project sponsored by the US Department of Energy, Office of Electricity Delivery and Energy Reliability, including information about (i) the architecture of these integrated systems and (ii) expanded functions of two example DMS applications to accommodate DERs: volt-var optimisation and fault location, isolation, and service restoration. In addition, the relevant DER group functions necessary to support communications between the DMS and a microgrid controller in grid-tied mode are identified.
\end{abstract}

\section{Introduction}

Management of the electric distribution grid has been occurring without the expansion of distribution management system (DMS) functions to fully account for the impact of the proliferation of distributed energy resources (DERs) and microgrids on the existing networks. Currently, the DMS, DERs, distributed energy resources management systems (DERMS), and microgrid controllers (MCs) are managed as separate entities with minimal communication, let alone coordination, among them.

Fig. 1 shows the topology of a proposed integrated system with the DMS, DERMS, DERs, and microgrids working together along with other management systems, including demand response automation systems (DRAS), a geographic information system (GIS), and an outage management system (OMS).

\subsection{DMS applications}

A DMS consists of an array of functions to meet different control objectives [1]. To operate efficiently, the DMS distributes management of the electric distribution system to different applications that perform specific functions. Depending on the application, objective realisation may involve monitoring the present state and forecasting the future state of the distribution system and/or selecting relevant values for a number of control settings. Currently, the DMS applications are able to adjust the control set points of traditional distribution equipment (load tap changers, line regulators etc.). The next generation of DMS will need to incorporate new grid-support capabilities offered by groups of DERs connected to the network. Microgrids, when functioning in grid-tied mode, are an example. Integrating these new DER group capabilities to realise system-level objectives will require DMS applications to communicate and exchange messages with these groups of DERs.

Numerous applications that are common among different DMS implementations handle the decisions and processes that are necessary to ensure that the electric distribution system operates efficiently, reliably, and safely [2].

These DMS applications can be grouped into three categories (Table 1) based on their objectives and roles in the electric distribution system:

System adjustment applications: These applications keep the electric distribution system within defined operating boundaries (e.g. voltage, current and power factor).

Operational (protection/reliability) applications: These applications reconfigure the connected electric distribution system to reduce system interruptions and change protection schemes when the circuit configuration is altered.

Situational awareness applications: These applications supply the system operators, system adjustment applications, and operational applications with actionable information to make decisions and perform adjustments. Situational awareness applications do not directly perform actions on distribution system equipment.

This paper focuses on the volt-var optimisation (VVO) and fault location, isolation, and service restoration (FLISR) applications as examples of the system adjustment and operational application categories.

\section{Architectural considerations}

Different configurations are possible to integrate a DMS with DERMS and MCs depending on the specific DMS solution selected by the utility. Some DMS software packages may integrate multiple systems into a DMS package, which is called an advanced DMS (ADMS) or an integrated DMS. Fig. 2 shows an example of this type of configuration with an integrated OMS and a common database. The ADMS communicates via supervisory control and data acquisition with field devices and via a utility enterprise integration bus to other applications such as a GIS, customer information system (CIS) and meter data management system (MDMS). Possible future interfaces with aggregators are also shown. 


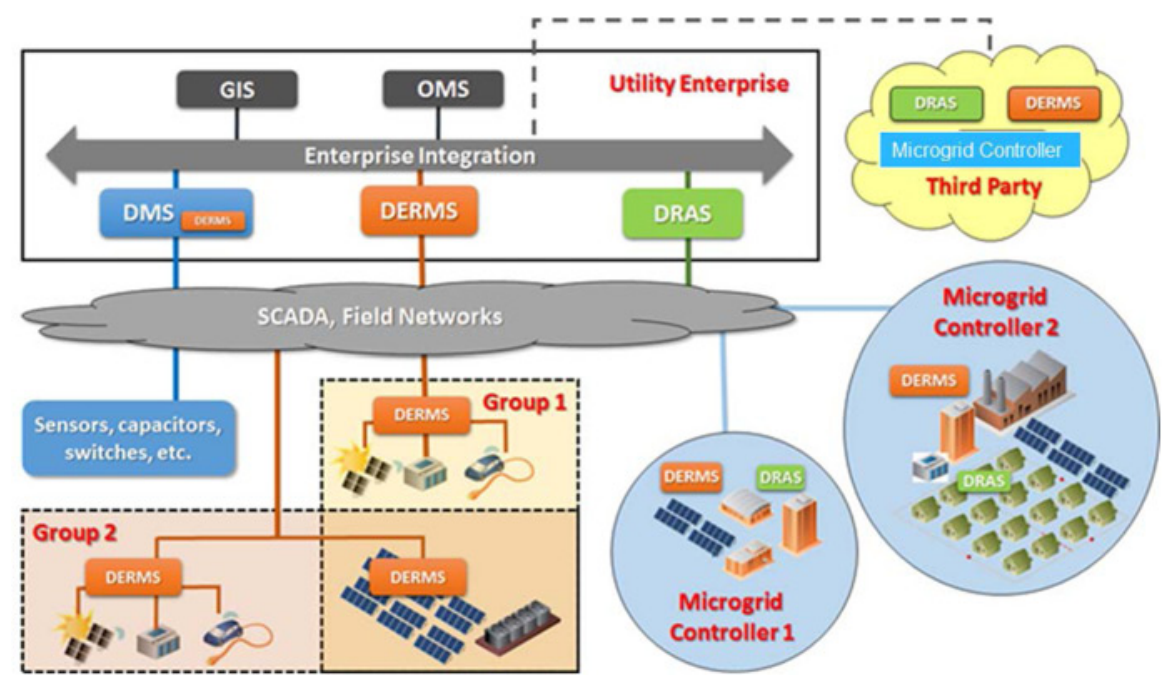

Fig. 1 Overview of an integrated electric distribution system. Source: EPRI 2016

Table 1 DMS applications by category

\begin{tabular}{|c|c|c|c|}
\hline \multirow[t]{2}{*}{ DMS application } & \multicolumn{3}{|c|}{ Categories } \\
\hline & $\begin{array}{c}\text { System } \\
\text { adjustment } \\
\text { (dispatch, energy } \\
\text { efficiency, load - } \\
\text { generation) }\end{array}$ & $\begin{array}{c}\text { Operational } \\
\text { (protection/ } \\
\text { reliability) }\end{array}$ & $\begin{array}{l}\text { Situational } \\
\text { awareness }\end{array}$ \\
\hline $\begin{array}{l}\text { volt-var } \\
\text { optimisation (VVO) }\end{array}$ & $\checkmark$ & - & - \\
\hline $\begin{array}{l}\text { conservation } \\
\text { voltage reduction }\end{array}$ & $\checkmark$ & - & - \\
\hline $\begin{array}{l}\text { power factor } \\
\text { correction }\end{array}$ & $\checkmark$ & - & - \\
\hline load balancing & $\checkmark$ & - & - \\
\hline phase balancing & $\checkmark$ & - & - \\
\hline online power flow & - & - & $\checkmark$ \\
\hline $\begin{array}{l}\text { contingency } \\
\text { analysis }\end{array}$ & - & - & $\checkmark$ \\
\hline $\begin{array}{l}\text { fault location, } \\
\text { isolation, and } \\
\text { servicer restoration } \\
\text { (FLISR) }\end{array}$ & - & $\checkmark$ & - \\
\hline $\begin{array}{l}\text { emergency load } \\
\text { shedding }\end{array}$ & $\checkmark$ & - & - \\
\hline $\begin{array}{l}\text { switch order } \\
\text { management }\end{array}$ & - & $\checkmark$ & - \\
\hline $\begin{array}{l}\text { short term load } \\
\text { forecast }\end{array}$ & - & - & $\checkmark$ \\
\hline $\begin{array}{l}\text { short term } \\
\text { generation forecast }\end{array}$ & - & - & $\checkmark$ \\
\hline demand response & $\checkmark$ & - & - \\
\hline $\begin{array}{l}\text { short circuit } \\
\text { analysis }\end{array}$ & - & - & $\checkmark$ \\
\hline voltage correction & $\checkmark$ & - & - \\
\hline $\begin{array}{l}\text { predictive fault } \\
\text { location }\end{array}$ & - & - & $\checkmark$ \\
\hline $\begin{array}{l}\text { optimal network } \\
\text { reconfiguration }\end{array}$ & $\checkmark$ & - & - \\
\hline $\begin{array}{l}\text { dynamic equipment } \\
\text { rating }\end{array}$ & - & - & $\checkmark$ \\
\hline
\end{tabular}

\subsection{Architecture variations with DERMS and microgrids}

The overall architecture for integrating a DMS with DERs, DERMS, and MCs varies based on circuit topology, utility operation practices, and connected distributed generation. A DMS can communicate with DERs in three different ways, and a combination of these may be present in a particular system, as illustrated in Fig. 1:

- The DMS communicates directly with individual DERs. In this case, the DMS obtains the device-level measurements from individual DERs, predicts future output power, and makes the measurements and predictions available to all the DMS functions to enhance performance.

- The DMS communicates with DERs through a DERMS. In this case, the DMS can obtain the device-level measurements from the DERMS and perform output power prediction; or the DERMS can predict future DER output power and supply that information to the DMS.

- The DMS communicates with a MC. In a system where microgrids are present, an MC is used to manage the DERs within it. Similar to the case with DERMS, the prediction of output power can be done by either the MC or the DMS. It is also possible for the MC to communicate through a DERMS to the DERs.

The architecture for integrating a DMS with MCs and DERMS needs to be considered in the context of the specific DMS applications that will interact with these systems. Additional functionalities should reside in each of the different controllers to enable these interactive DMS functions.

\subsection{VVO application}

As a core DMS application, VVO has been selected as an example system adjustment application. Some VVO control objectives include maintaining an acceptable feeder voltage profile and reactive power flows during normal operation.

VVO can play an important role in the support of higher penetrations of DERs; therefore, the impact of DER penetration on the VVO application needs to be considered. When a DER with a smart inverter is connected to the distribution system, possibly within a microgrid, the reactive power and voltage at its terminal may be continuously adjustable within certain ranges through control set points. Increasing penetrations of these smart DERs introduce new challenges to the VVO algorithms because they need to accommodate mixed control variables, both discrete and continuous. This requires software modifications at appropriate control points.

2.2.1 Communications: In the case of VVO, a DERMS or MC may send the forecasted/scheduled DER generation to the DMS. The VVO application in the DMS would determine the overall volt-var droop control curve or power factor set point for the DER group or microgrid and send those to the DERMS/MC, which, in turn, may send volt-var droop control curve settings or power factor set points to each individual smart inverter. If the DERs are dispatchable, the real power set points may be sent as well, if applicable. If a microgrid is present, the $\mathrm{MC}$ may send the forecasted active power and reactive power support capabilities to 


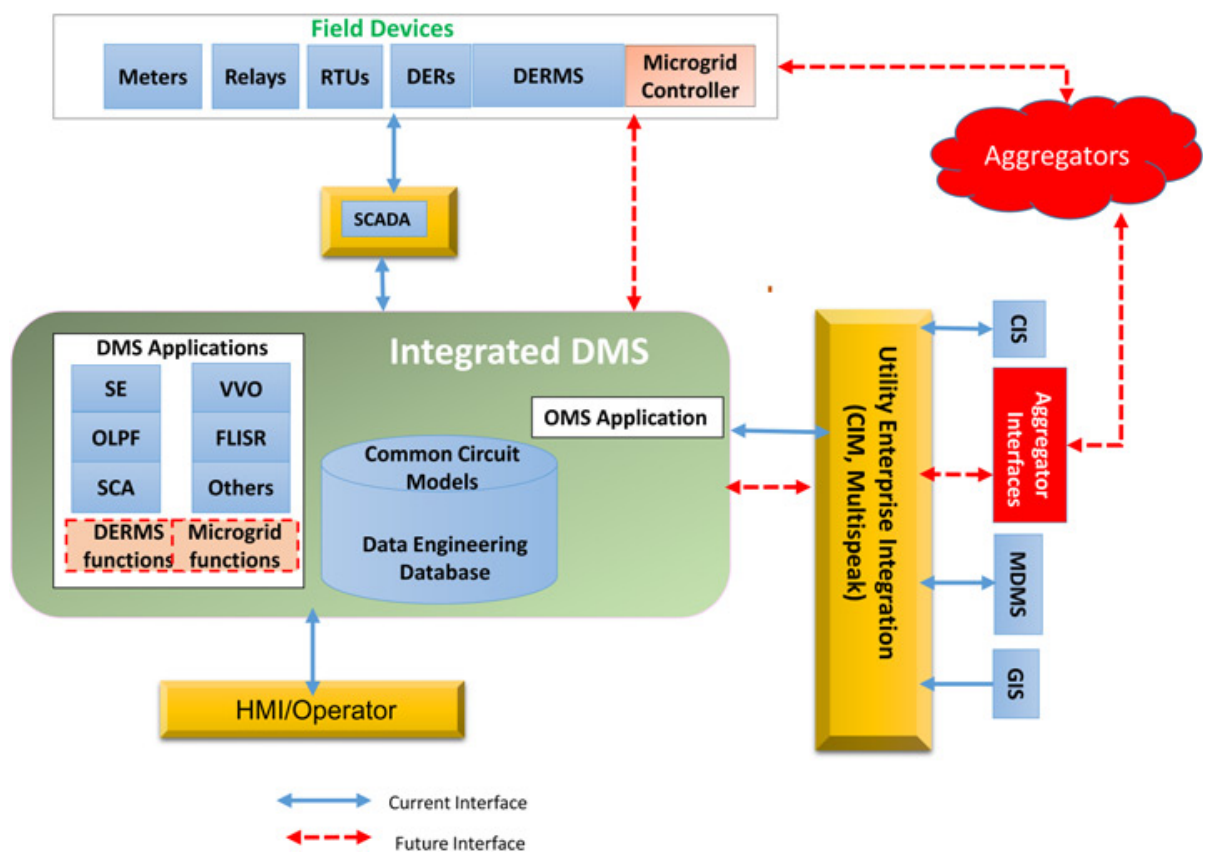

Fig. 2 Configuration of an integrated DMS system

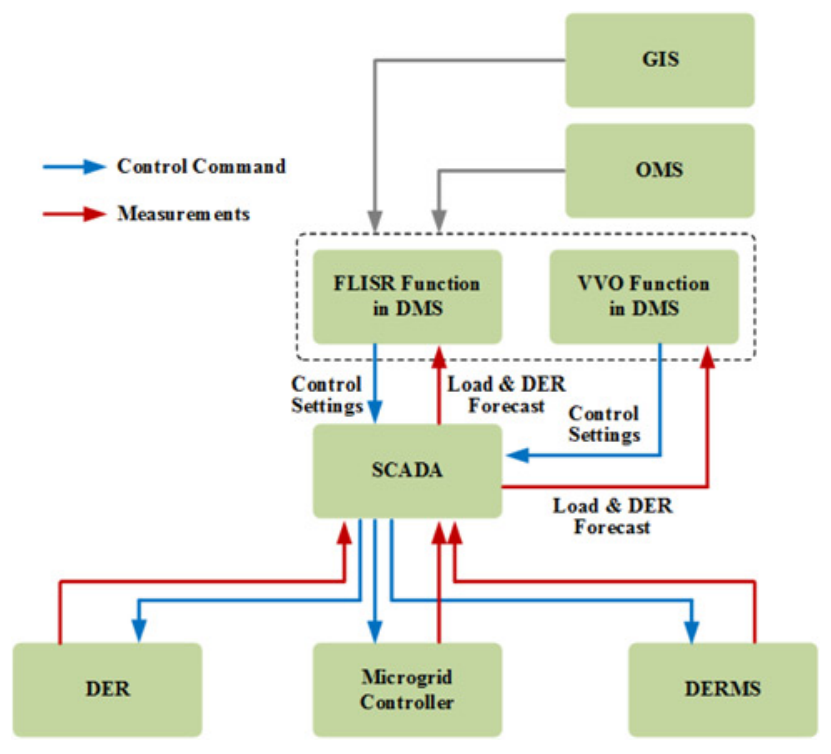

Fig. 3 Data flow among controllers

the DMS VVO application. The VVO application may then send power factor or voltage set points to each individual MC.

A more detailed definition and description of the specific signals requires a systems approach-based analysis that includes distributing the VVO functionality, ascertaining where the actual controls reside, and developing the interfaces among these actors. This information flow is illustrated in Fig. 3.

\subsection{FLISR application}

FLISR is an example of an operational (reliability/protection) application. FLISR is a suite of applications that are used to mitigate the impact of permanent faults on distribution systems and ensure a high level of overall system reliability.

The objectives of FLISR include automatic detection of permanent faults (faults that are not self-clearing) on the distribution feeder, determination of the approximate location of the fault (i.e. the faulted section of the feeder that is bounded by two or more automated feeder switches), and isolation of the faulted section of the feeder and network reconfiguration to restore loads that are downstream of the isolated faulted section. FLISR is designed to prevent service restoration when alternate circuits do not have enough capacity to carry additional load. Since alternate circuit feeds are used for restoration, FLISR influences other circuits on the system due to switching.

The integration of increasing penetration levels of DERs has changed the operational principle of the FLISR application because of the following factors:

- intermittence and power variation induced by non-dispatchable DERs,

- bidirectional power flow that influences the conventional protection schemes,

- impact of MCs that aggregate local DERs and loads.

2.3.1 Communications: In the context of the FLISR function, the location, status, and current state of the protective devices during a fault condition should be utilised to isolate the distributed DER feeding into the fault.

In the case of FLISR, data flow that is distributed among various actors - such as DMS, OMS, DERMS, and/or a MC - is required to manage normal, faulted, islanded, or non-islanded states of the microgrid or distribution system segments that are at fault. A fault inside the microgrid may be isolated by opening the breaker at the point of interconnection (POI) of the microgrid. A fault on the feeder connecting the microgrid also requires the microgrid to be transitioned to an islanded mode to prevent back feeding the fault and to minimise disruption to the microgrid loads.

The data flow among different actors, as shown in Fig. 3 should be further studied. For device-level components, it is necessary to coordinate the settings of protective relays with upstream relay settings. Finally, the FLISR application in the DMS should be able to obtain the predicted output power of DERs or the corresponding microgrids so that the alternative feeder can be accurately selected. 


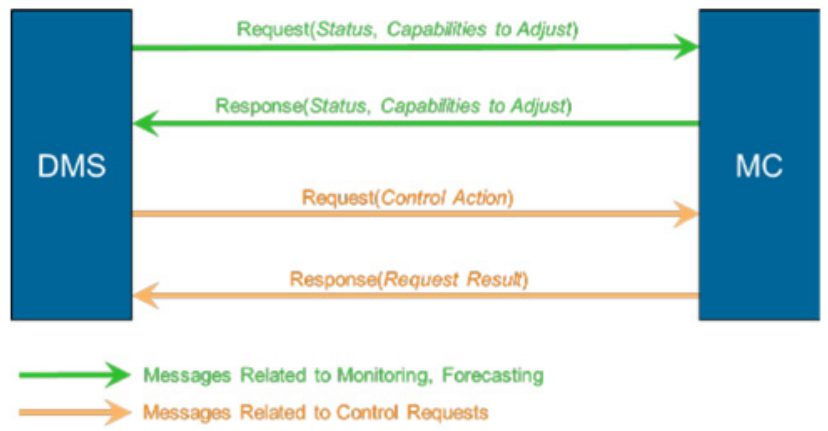

Fig. 4 Approach to identifying relevant DMS-MC messages for each DMS application

\section{Messages between DMS and MC}

The interaction between a DMS and a MC is considered in the following section. These examples illustrate the different messages that need to be exchanged to implement interactive VVO and FLISR applications. Fig. 4 presents a generic version of this interaction wherein the DMS could send requests and receive responses to and from the $\mathrm{MC}$ for two types of messages: monitoring/forecasting and control set points.

A starting point to structure the messages exchanged between the DMS and the MC is to identify which variables are of interest to the DMS and how these variables are to be used - e.g. for monitoring, forecasting, or control requests. The next step consists of identifying the relevant DER group functions that enable the variables of interest to be monitored, forecasted, and/or controlled as needed. Since 2012, the Electric Power Research Institute (EPRI) has facilitated a focus group of industry experts who are developing a library of standard DER group functions to monitor and manage groups of DERs [3]. Group functions offer a standardised way to read or report the status and capabilities of a DER group (such as a grid-tied microgrid) and send grid-support requests to the entity controlling the group (in this example, the MC), as shown in Fig. 5.

Each DER group function has its own set of standard messages as defined in [3]. The DER group functions that enable the two types of messages - control and forecasting/monitoring - for the two applications are identified below:

\subsection{Status monitoring and forecasting at the $\mathrm{POI}$}

Both DMS applications considered in this paper, VVO and FLISR, need to monitor the present and forecasted status of a number of variables defined at the microgrid POI. In addition, VVO needs to know the extent of the MC's capabilities to support the grid at the POI. With this information, the VVO can decide which control set points that are available from the MC can be incorporated into the VVO algorithm. Two DER group-level functions defined in [3], Status Monitoring of DER groups and DER group output forecasting, enable the DMS to obtain this information from the MC.

\subsection{Sending DMS control requests for grid support}

VVO differs from FLISR because, in addition to monitoring the microgrid status and capabilities, VVO requires a mechanism to

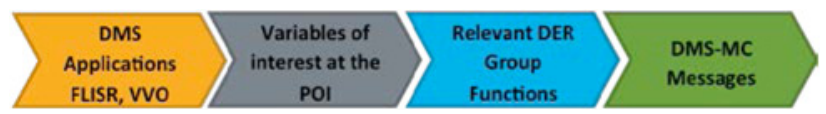

Fig. 5 Messages between a DMS and MC enabling DMS applications to (i) monitor and forecast microgrid status and capabilities and (ii) enable requests for support send requests for grid support to the MC. Based on the microgrid capabilities available, the VVO algorithm can select for each variable either a set point (dispatch) or an operating range (boundaries) to send to the MC. The DER group functions enabling the DMS to specify either a set point or a boundary to

Table 2 Relevant DER group functions identified for the two DMS applications considered

\begin{tabular}{llc}
\hline DER group function & VVO & FLISR \\
\hline status monitoring of DER groups & $\bullet$ & - \\
DER group output forecasting & $\bullet$ & - \\
DER group maximum real power limiting & $\bullet$ & - \\
real power dispatch of DER groups & $\bullet$ \\
reactive power dispatch of DER groups & $\bullet$ \\
\hline
\end{tabular}

Table 3 Messages sent by a DMS to a MC for the status monitoring of DER groups requests

\begin{tabular}{|c|c|}
\hline Information name & Information description \\
\hline action identifier & $\begin{array}{c}\text { defines what is being requested: DER group status } \\
\text { request }\end{array}$ \\
\hline $\begin{array}{l}\text { timing of status } \\
\text { request }\end{array}$ & $\begin{array}{l}\text { defines whether the request is for latest available } \\
\text { (MC can provide immediately) or refreshed status } \\
\text { (MC needs to update information first) }\end{array}$ \\
\hline & $\begin{array}{l}\text { the unique identifier of the DMS that created } \\
\text { (or last modified) this request. }\end{array}$ \\
\hline microgrid ID & $\begin{array}{l}\text { the unique identifier for the microgrid for which the } \\
\text { status is being requested }\end{array}$ \\
\hline quantity & $\begin{array}{c}\text { the quantity requested - e.g. active power, real } \\
\text { power and apparent power }\end{array}$ \\
\hline
\end{tabular}

Table 4 Response sent by a MC to a DMS for the status monitoring of DER groups requests

\begin{tabular}{lc}
\hline Information name & Information description \\
\hline microgrid ID & $\begin{array}{r}\text { the unique identifier of the microgrid for which } \\
\text { status was requested } \\
\text { DMS ID }\end{array}$ \\
$\begin{array}{l}\text { the unique identifier of the DMS that created } \\
\text { (or last modified) this request } \\
\text { indicator(s) } \\
\text { variable requested } \\
\begin{array}{l}\text { n1 } \\
\text { variable requested } \\
\text { \#2 }\end{array}\end{array}$ & $\begin{array}{c}\text { indication of whether or not, or to what extent the } \\
\text { command could be accommodated } \\
\text { present value of variable }\end{array}$ \\
\hline
\end{tabular}

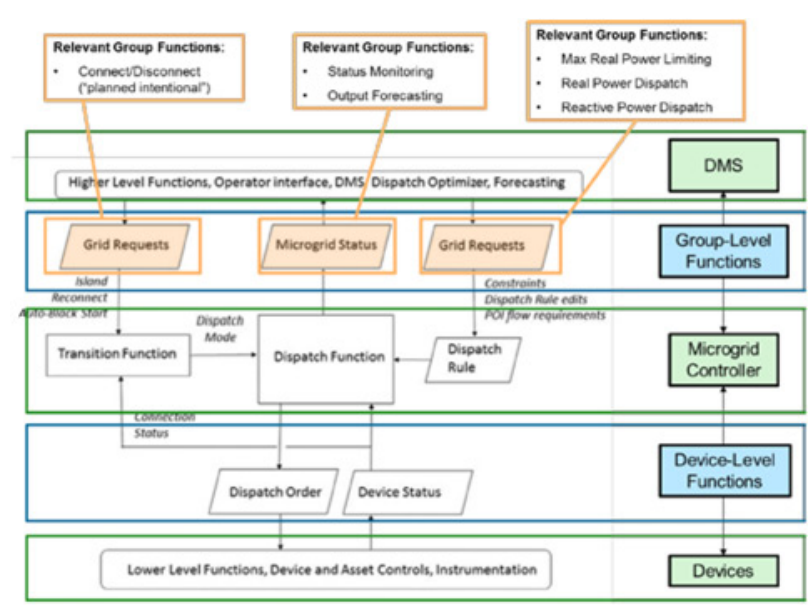

Fig. 6 Relationships among the IEEE 2030.7 transitions and dispatch functions and EPRI's group-level and device-level functions. Figure adapted from IEEE 2030.7 
stay within the variables include DER group maximum real power limiting, real power dispatch of DER groups, and reactive power dispatch of DER groups.

\subsection{Messages exchanged for DER group functions identified as relevant}

Table 2 summarises the DER group functions relevant to the VVO and FLISR applications. The detailed messages supporting the implementation of these can be found in [3]. Tables 3 and 4 show the messages exchanged between the MC and DMS when calling the status monitoring of DER groups function.

Fig. 6 illustrates the interactions taking place among the DMS, $\mathrm{MC}$, and DER assets. It connects the approach presented in this section to the concepts defined in draft standard IEEE 2030.7 (specifications of microgrid controllers).

\section{Conclusions}

This paper describes the architecture of an integrated electric distribution system management approach based on the coordinated operation of a DMS, MCs, DERMS, and DERs. The relevant DER group functions, communications, and the interactions necessary to support the implementation of two selected DMS applications, VVO and FLISR, are identified. The information presented in this paper is based on prior work on DER group functions and an architecture and message development effort currently underway titled 'Structuring a Demonstration Project to Integrate DER, Microgrid, DERMS, and DMS' that is funded by the US Department of Energy, Office of Electricity Delivery and Energy Reliability.

\section{References}

1 Wang, J.: 'ADMS for grid modernization: DMS functions' (Argonne National Laboratory, Lemont, IL, 2015), ANL/ESD-15/17

2 Wang, J., Lu, X., Reilly, J., et al.: 'ADMS for grid modernization: high-level use cases for DMS' (Argonne National Laboratory, Lemont, IL, 2015), ANL/ESD-15/ 18

3 EPRI: 'Common functions for DER group management' (Palo Alto, CA, 2016, 3rd edn.), 3002008215 